\title{
SURGICAL MANAGEMENT OF FRACTURE BOTH BONES OF FOREARM WITH LOCKING COMPRESSION PLATE IN ADULT PATIENTS
}

\author{
KRISHNA BHARGHAVA VEM ${ }^{1 *}$, SHIVA KUMAR CHALLA ${ }^{2}$, SRINIVAS MURTHY ${ }^{1}$
}

\begin{abstract}
${ }^{1}$ Department of Orthopaedics, Apollo Institute of Medical Sciences and Research, Hyderabad - 500 033, Telangana, India. ${ }^{2}$ Department of Orthopaedics, Gandhi Medical College and Hospital, Musheerabad, Secunderabad - 500 003, Telangana, India. Email: krishnabr@live.in
\end{abstract}

Received: 26 June 2018, Revised and Accepted: 07 August 2018

\section{ABSTRACT}

Objective: The objective of this study was to assess the time taken for surgery, tourniquet time, and clinical outcome in diaphyseal fractures of both forearms managed with locking compression plate (LCP).

Methods: Patients underwent open reduction and internal fixation with 3.5-mm LCP. Proximal radius was approached by dorsal Thompson incision and middle and distal radius by volar henry approach. A minimum of 6 cortices were engaged with screw fixation in each fragment. Ulna was approached directly over the subcutaneous border.

Results: Of 20 patients, 70\% were men; fracture was the most common in second and third decades of life. Road traffic accident (50\%) and fall (40\%) were the main causes. Fractures at mid-diaphysis (70\%) and transverse/short oblique (72.5\%) were the most common. Closed head injury, unilateral pubic bone fracture, olecranon fracture, fracture of both bones of leg, and ipsilateral fracture shaft humerus were the associated injuries. There was no intraoperative complication. Superficial infection $(\mathrm{n}=01)$ and transient posterior interosseous nerve injury, in the immediate postoperative period $(n=01)$, were the reported complications. Average time for surgery was $77 \mathrm{~min}$ (60-90 min) and that for tourniquet was $54 \mathrm{~min}$ (40-60 min). All had complete union in $<6$ months; $80 \%$ had healing in $<4$ months. $20 \%$ healed in $4-6$ months. Excellent and satisfactory results were seen in $85 \%$ and $15 \%$ patients, respectively.

Conclusion: The 3.5-mm LCP, when applied properly, yields excellent outcome in fractures of forearm bones. The use of tourniquet, separate incisions for radius and ulna, and preservation of the natural curves of radius will reduce complications. Clinical outcome in terms of healing and union is excellent.

Keywords: Diaphyseal fractures, Locking compression plate, Operation time, Tourniquet time.

(c) 2019 The Authors. Published by Innovare Academic Sciences Pvt Ltd. This is an open access article under the CC BY license (http://creativecommons. org/licenses/by/4. 0/) DOI: http://dx.doi.org/10.22159/ajpcr.2019.v12i3.28801

\section{INTRODUCTION}

Higher prevalence of malunion and non-union associated with the fractures of the forearm makes the management challenging and complicated, but improvised surgical techniques have revolutionized the management and help in overcoming these challenges [1]. Lessons from the clinical applications and compression plating and internal fixation lead to the development of an implant system that combines different therapeutic modalities that help in regaining length, positioning, and alignment of the fractured bones.

Locking compression plate (LCP) is a product of latest plating techniques, designed to have advantages of performing surgery with a minimal length of incision, preserving and retaining blood supply to the bone and adjacent soft tissues. In addition, providing stability at the fracture site is also an added advantage, a disappointing factor with other techniques. Reddy et al. [2] reported that the use of LCP in forearm fractures is an ideal procedure as stable fixation along with early union is ensured.

LCP has features of both LC-dynamic compression plate (DCP) and a point contact fixator $[3,4]$ as it uses screw heads that are conically threaded on the undersurface and create an angular stable plate screw device. This type of plate fixation relies on the threaded plate-screw interface to lock the bone fragments in position and does not require friction between the plate and bone as in conventional plating. This method is postulated to result in better bone healing due to a reduction in associated complications (infections, bone resorption, delayed/nonunion, and secondary reduction rate) [5] but with limited data to prove its efficacy and comparison with other plates in the management of forearm fractures [6-9]. Available data, though limited, show that the use of LCP in Indian population yield good outcome [10-13] can be considered as a treatment option for forearm fractures [14].

\section{METHODS}

This prospective study was conducted by the Department of Orthopedics of a tertiary care teaching hospital in India from November 2014 to August 2017, after obtaining the Institutional Ethics Committee's approval, and conducted as per the ethical standards. Prospective patients were screened only after obtaining the written informed consent, and those met the inclusion and exclusion criteria were included in the study. Patients who aged $>18$ years, with diaphyseal fractures of both forearms, and fit for surgery were included while those with compound fractures and spiral/segmental fractures of forearm bones, unwilling, and medically unfit for surgery were excluded from the study. We evaluated the use of LCPs in fractures of forearm bones in adult Indian patients and assessed the time taken for surgery, tourniquet time, and clinical outcome.

A detailed medical and surgical history was elicited from the hospitalized patient and/or attendants to know the mechanism of injury and the severity of trauma which were assessed clinically to evaluate their general condition and local injury. Clinical examination was done to rule out fractures at other sites. Local examination of injured forearm included assessment of the extent of swelling, deformity, and loss of function, in addition to any nerve injury. Abnormal mobility, crepitus, and shortening of the forearm, if any, were noted. Distal vascularity 
was assessed by radial artery pulsations, capillary filling, pallor, and paraesthesia at fingertips.

Radiographs of the radius and ulna (anteroposterior and lateral views) were obtained. The elbow and wrist joints were included in each view. The limb was then immobilized in above elbow Plaster of Paris slab with a sling.

Patients were taken for surgery after routine investigations after assessing their medical fitness.

A pneumatic tourniquet was applied. Proximal radius was approached by dorsal Thompson incision and volar Henry approach was used for the middle and distal radius. A narrow $3.5 \mathrm{~mm}$ LCP was used, and a minimum of six cortices was engaged with screw fixation in each fragment. Ulna was approached directly over the subcutaneous border. The bone which was less comminuted and more stable was fixed first, and later, the other bone was fixed [15]

After identifying the fracture, ends were cleaned without elevating the periosteum. With the help of reduction clamps, the fracture was reduced and held in position. The plate was then applied after contouring, if required. A plate of at least 6 holes was chosen, and longer plates were used for spiral, segmental, and comminuted fractures. For upper-third radial fractures, plate fixation on dorsal aspect was considered. For the middle-third, the plate was fixed dorsolaterally, and for distal radial fractures, the plate was fixed on the volar aspect (Fig. 1). In ulnar fractures, plate was applied over the posterior surface of ulna [16].

A drill sleeve for locking screw was fixed in the hole, near the fracture site, and 2.7- $\mathrm{mm}$ drill bit was used to drill both the cortex of the bone, the sleeve was removed, and the screw length was measured with a depth gauge. A 3.5-mm locking screws were inserted (Fig. 1). The locking screws are self-tapping; hence, tapping of the screw hole was not required.

After adaptation of the fragments, a screw hole for axial compression was drilled in the fragment that formed an acute angle near the plate. Here, the load guide was used with the arrow pointing toward the fracture line to be compressed. At this position, a lag screw was inserted for axial compression.

The lag screw was applied subsequently over the drilling $(3.5 \mathrm{~mm})$, near cortex to create a gliding hole. The lag screw and remaining screws were inserted (Fig. 1). Once stable, fixation was achieved and hemostasis secured meticulously, the wound was closed in layers over a suction drain, and sterile dressing was applied.

Postoperatively, all patients were advised to apply a crepe bandage over the affected forearm and arm pouch. Patients were instructed to keep the limb elevated and move their fingers and elbow joint. A suction drain was removed after 24-48 h. The wound was inspected after 3-4 days, postoperatively. Antibiotics and analgesics were given to the patient until the time of suture removal. Suture/staples were removed on $10^{\text {th }}$ post-operative day, and check X-ray in anteroposterior and lateral views was obtained.

Patients were discharged after suture/staple removal with the forearm in arm pouch and advised to perform shoulder, elbow, wrist, and finger movements. Patients were advised not to lift any heavy weight or exert the affected forearm.

All were followed up at monthly intervals for first 3 months and evaluated as per Anderson et al. scoring system [17]. Elbow and wrist movements were noted and fracture union was assessed radiologically.

The fracture was considered as united when there were no subjective complaints and the fracture line was invisible radiologically and

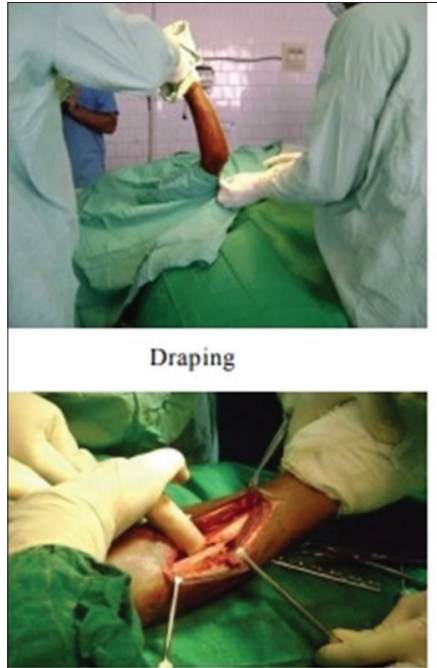

Fracture fragments exposed And reduced

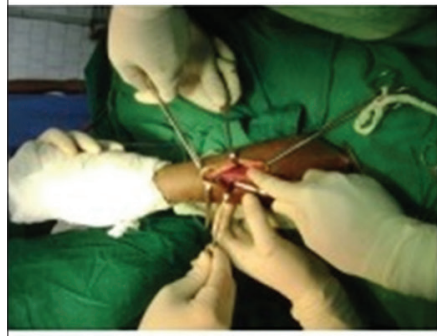

Subcutaneous approach for ulna

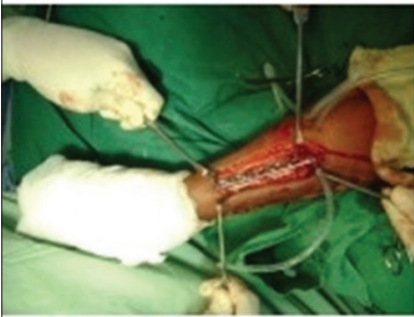

Fixed with $3.5 \mathrm{~mm} \mathrm{LCP}$

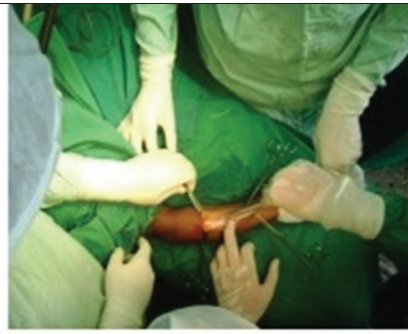

Volar approach for radius

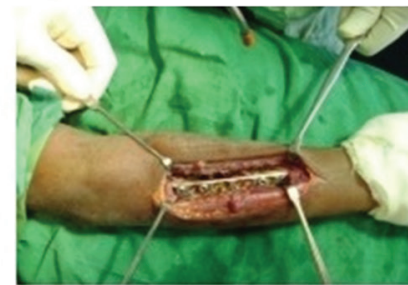

Fixed with 6 holed $3.5 \mathrm{~mm}$ LCP

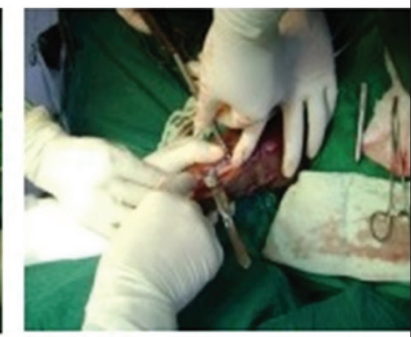

Bone exposed and reduced

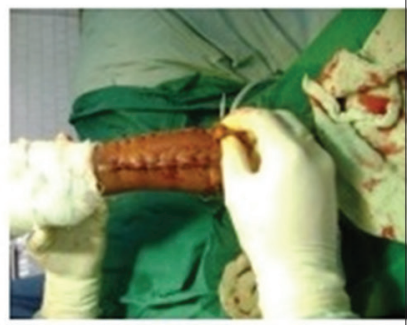

Wound closed

Fig. 1: Various steps of surgical technique used in fixing forearm fracture

designated as united in the presence of periosteal callus bridging the fracture site and extensions of trabeculation across the fracture line. The fracture that healed after 6 months without an additional operative procedure was considered as a delayed union. Fractures that did not unite after 6 months or that needed an additional operative procedure to unite were considered as non-union

\section{Statistical analysis}

Data were captured on Microsoft Excel Worksheets (2007) and analyzed. Results were expressed as mean, frequency, and range. Tables and figures were used as appropriate.

\section{RESULTS}

We included 20 patients who met inclusion criteria and underwent open reduction and internal fixation with $3.5 \mathrm{~mm}$ LCP. There were $14(70 \%)$ men and six (30\%) women.

Mean age was 33.5 years, ranging from 18 to 55 years, and the fracture was the most common in second and third decades of life (Table 1). 
Road traffic accidents $(n=10,50 \%)$ and fall $(n=08,40 \%)$ were the main causes and only two $(10 \%)$ patients had a history of assault. There were $14(70 \%)$ and six (30\%) patients with left and right forearm fracture, respectively. All fractures were closed injuries.

\section{Level and type of fracture}

Fractures at mid-diaphysis $(n=14,70 \%)$ was the common, and three $(15 \%)$ patients each had proximal third and lower third fracture of both bones of the forearm (Fig. 2)

Transverse/short oblique $(72.5 \%)$ was the most common type of fractures (Table 2).

Five (25\%) had associated injuries; closed head injury, unilateral pubic bone fracture, olecranon fracture, fracture of both bones of the leg, and ipsilateral fracture shaft humerus were seen in these patients.

Fourteen were operated under general anesthesia, and for six patients, brachial block was used. Dorsal Thompson approach $(n=05,25 \%)$ and volar Henrys approach $(n=15,75 \%)$ for radius were used. Ulna was approached subcutaneously. Pneumatic tourniquet was used in all. Follow-up ranged from 5 months to 24 months

There was no intraoperative complication. Two had post-operative complications; one patient developed superficial infection managed with appropriate antibiotics as per culture and sensitivity report.

One patient with proximal radius fracture developed transient posterior interosseous nerve injury, in the immediate post-operative period. This patient was treated with a static cock-up splint which recovered in a span of about $1 \frac{1}{2}$ months.

The duration of surgery for fixation of both bones' forearm ranged from 60 to $90 \mathrm{~min}$, with an average time of $77 \mathrm{~min}$. The tourniquet time ranged from 40 to $60 \mathrm{~min}$, with an average time of $54 \mathrm{~min}$

All $(100 \%)$ had a complete union in $<6$ months with $16(80 \%)$ healing in $<4$ months and 04 requiring 4-6 months.

Seventeen $(85 \%)$ patients showed excellent results, and three (15\%) had satisfactory results with the procedure.

Figs. 2 and 4 show the pre- and post-operative radiological features, and Figs. 3 and 5 show the functional outcome in patients.

\section{DISCUSSION}

Fracture, both bones of forearm, presents a formidable challenge to the orthopedicians as the various muscle forces acting on the fracture tend to displace it. Hence, to provide the functional rehabilitation of

Table 1: Age-wise distribution of the study population

\begin{tabular}{ll}
\hline Age (years) & n (\%) \\
\hline $18-20$ & $2(10)$ \\
$21-30$ & $8(40)$ \\
$31-40$ & $6(30)$ \\
$41-50$ & $3(15)$ \\
$51-60$ & $1(05)$ \\
Total & $20(100)$ \\
\hline
\end{tabular}

Table 2: Type of forearm fractures

\begin{tabular}{lll}
\hline Type of fracture & Radius & Ulna (\%) \\
\hline Transverse/short oblique & 14 & $15(72.5)$ \\
Comminuted & 6 & $4(25)$ \\
Segmental & - & $01(2.5)$ \\
Total & 20 & $20(100)$ \\
\hline
\end{tabular}

the upper limb, anatomic reduction and rigid fixation are mandatory. This is achieved by open reduction and internal fixation with DCP and screws [18]. Fracture management is a dynamic procedure advancing since the first introduction of internal fixation in 1886 by Hansmann in Hamburg[19]. Improvements were sought to achieve stable internal fixation and stable bone-implant connection; early functional mobilization led to the use of bridging plates. The use of bridging plates is thought to be associated with undisturbed fracture zone, providing relative stability and secondary healing with callous formation meeting the treatment objectives. In addition, this procedure is not associated with devascularization of fracture fragments. Surgical adaptations are required for the application of LCP.

LCP, a fracture correction procedure, is rapidly gaining popularity among orthopedicians due to its comparable efficacy and clinical outcome with the conventional implants but with advantages. Sommer et al. [20] considered this technique as more technically advanced and mature yielding better clinical outcome of good-to-excellent grade.

In our study, the fracture was more common in the second and third decades, with an average age of 33.5 years (18-55 years). Similar observation but with a higher mean age was reported by Burwell and Charnley [6,21-24]. Male preponderance is a well-documented feature of forearm fractures [22-25], and our study is in support of this. Road

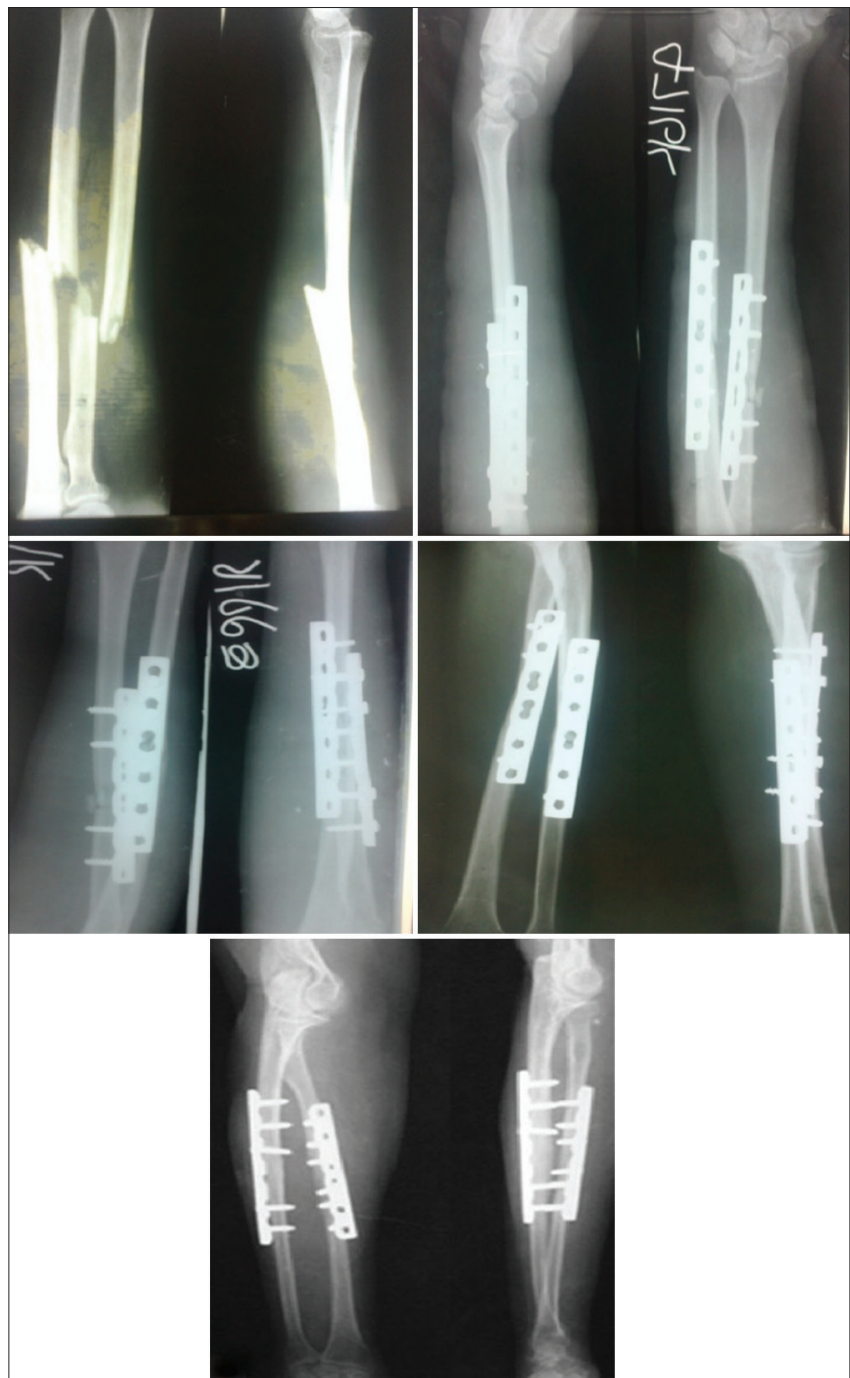

Fig. 2: Radiological images showing a fracture in the preoperative period, position of locking compression plate in immediate postoperative, and union during follow-up 


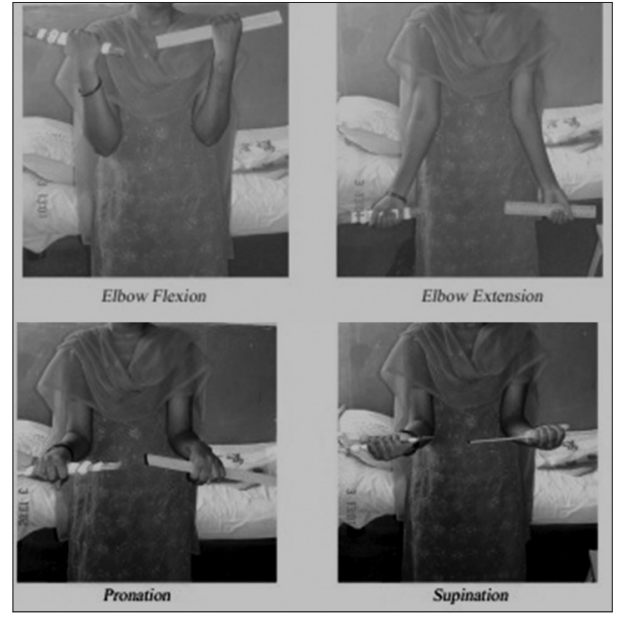

Fig. 3: Functional outcome. Patient demonstrating various movements

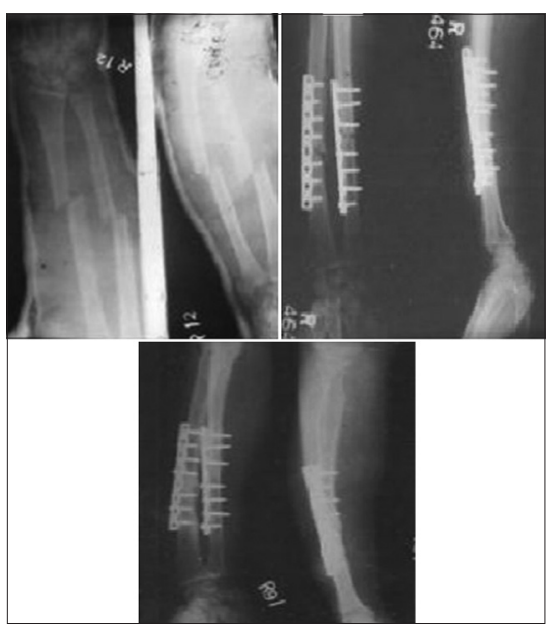

Fig. 4: Radiological images showing a fracture in the preoperative period, position of locking compression plate in immediate post-operative, and union during follow-up
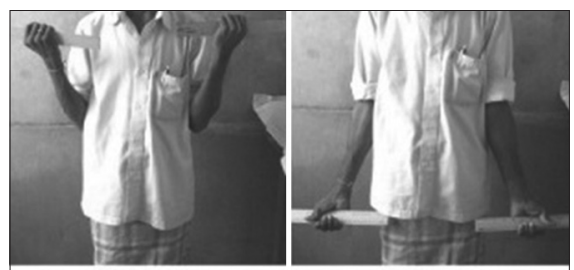

Elbow Flexion

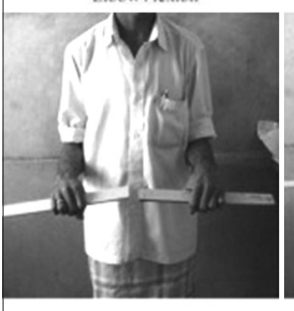

Elbow Extension

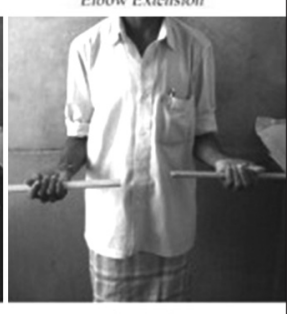

Pronation

Supinatiou

Fig. 5: Functional outcome. Patient demonstrating various movements

traffic accidents, fall, direct blow, and gunshot injuries have been reported as the most common causes of forearm fractures [22,26,27] Road traffic accidents $(5.0 \%)$, fall $(40 \%)$, and direct blow (assault)
$(10 \%)$ were the causes in our study. In addition, industrial accidents as a cause were reported by Moed et al. [22], but none of our patients had this as a cause.

Chapman et al. [24] noted comminuted fractures (53\%) as the most common type among forearm fractures while our patients had a transverse/short oblique $(72.5 \%)$ as the most common. This could be due to low-velocity trauma in our patients. Middle third and distal third are the common sites of fractures, less frequent in proximal third; $[22-24,27,28]$ fractures of the middle third were common in our patients as well but the distal and proximal third were equally affected.

LCP has proven good healing record; Leung et al. [6] reported delayed union in only two $(2 / 32)$ of their patients, and there was no non-union. Our patients had an average union time of 11.85 weeks (8-20 weeks), with $100 \%$ union of both radius and ulna. Time taken for complete union is in accordance with that reported by Chapman et al. (12 weeks) [24], while shorter time has been reported by Anderson et al. (7.4 weeks) [17] and McKee et al. (10.7 weeks) [29] and longer time of 17 weeks by Leung et al. [6]. These authors have reported the rate of complete union of 97-98\% except Leung et al. (100\%).

Functional outcome was assessed based on the range of motion using Anderson et al. score as a tool. The range of motion was reported excellent (50.9\%), satisfactory (34.9\%), unsatisfactory (11.3\%), and failure (2.9\%) by Anderson et al. [17], while Chapman et al. [24] reported $36(86 \%)$ cases as excellent, $3(7 \%)$ satisfactory, $1(2 \%)$ unsatisfactory, and 2 (5\%) failure. Leung et al. [6] reported 98\% of cases as excellent and $2 \%$ of satisfactory results. We obtained excellent results in $85 \%$ and satisfactory results in $15 \%$ of our study population; our patients did not have unsatisfactory results and failure.

Infection, non-union, posterior interosseous nerve injury and radioulnar synostosis are the reported complications with the procedure $[17,24]$. Leung et al. [6] reported superficial infection in one patient and refracture after implant removal in two. We had two patients, one each with infection and posterior interosseous nerve injury immediately following surgery and were managed appropriately.

The duration of surgery ranged between 60 and $90 \mathrm{~min}$, with an average of $77 \mathrm{~min}$. The tourniquet time ranged from 40 to $60 \mathrm{~min}$, with an average of 54 min. Meena et al. [10] reported a mean time for surgery as $70.25 \mathrm{~min}$.

After LCP fixation, post-operative support, given in the form of arm pouch in most instances, can be discontinued after the soft tissues have healed and rapid return to full, painless motion which can be anticipated.

In the Indian scenario, a study by Prakash and Basanthi [30], Naik and Gunnaiah [31], and Muralidhar et al. [32] shown similar results as ours. Demographic profile reported in the study by Reddy et al. [2] was similar to our study; road traffic accident $(76.7 \%)$ was the common cause while fall was the other cause. Fractures of middle third (46.6\%) were the most common followed by the distal third $(36.6 \%)$ and proximal third. They report that all fractures were closed type and included both transverse and comminuted type. In contrast to ours, associated injuries were less $(25 \%$ vs. $3.3 \%)$. Mean union time was longer (14.1 \pm 1.3 weeks). The range of movement was full in $86.7 \%$ and $10 \%$ reported to have good movement; loss of $10^{\circ}$ pronation was reported in $3.3 \%$. None of our patients had any loss in the range of movement. Superficial infection $(3.3 \%)$ and posterior interosseous nerve injury (6.7\%) were reported as complications. Excellent (83.3\%) and satisfactory (16.7\%) were the outcomes. Demographic profile reported by Sharma et al. [12] is similar to that of our study; open fractures $(13.3 \%)$ and a high prevalence of multiple injuries (33.3\%) were not in line with our study. Similar to our study, this study reported complete union in all (though two had delayed union), and mean time taken for 
union is being 12.6 weeks (range $8-24$ weeks). Infection $(\mathrm{n}=01)$ and stiffness of elbow and wrist $(04 / 30)$ were the reported complications. In contrast to ours, the outcome was satisfactory in $73.3 \%$, excellent in $16.7 \%$, and unsatisfactory in $10 \%$ [12]. Meena et al. [10] too supported the application of this technique in Indian patients in terms of better outcome and time taken for the bone union. They achieved an excellent outcome in $90 \%$ and satisfactory in $10 \%$ of their study population. Meantime taken for the union was 12.8 weeks; two patients reported to have developed an infection.

We did not encounter implant specific problems in our patients; however, one has to be aware and careful to identify early in these patients. Fracture non-union may require a second operation. The surgeon has to aware that delayed union and fracture healing though reported less is an unignorable outcome.

As highlighted by Azboy et al. [33], outcome is dependent on the technique used and surgical skill, and we are in agreement with this. LCP application involves a technique of reduction, inserting a plate through a minimally invasive procedure and fixing the fractured fragments without disturbing the bone viability. Niemeyer and Südkamp [34] re-emphasize views of Sommer et al. [35] that the clinical expertise to understand the fracture and plate biomechanics and biological osteosynthesis is necessary for the management.

This procedure is not free from drawbacks; the surgeon has no tactile feedback as to the quality of screw purchase into the bone as he tightens the screw. As the screw locks in the plate, all screws abruptly stop advancing when the threads are completely seated in the plate regardless of bone quality. Current locking plate designs can be used to maintain fracture reduction but not to obtain it. The fracture must be reduced and limb alignment, and length and rotation must be set properly before placement off any locked screws. The inability of the surgeon to alter the angle of the screw within the hole and still achieve a locked screw is a problem that needs to be addressed. Any attempt to contour locked plates could potentially distort the screw holes and adversely affect screw purchase.

Small sample size and not comparing with other available surgical techniques prevent us from making a strong recommendation on the superiority of the technique or arrive at a confident conclusion.

Our study proves that LCP is an effective treatment option for those with the fracture of both forearm bones.

\section{CONCLUSION}

The 3.5-mm LCP, properly applied, is an excellent method for internal fixation of fractures of the forearm bone. The use of tourniquet, separate incisions for radius and ulna, and preservation of the natural curves of radius will lessen the rate of complications.

These fractures have to be fixed as early as possible and it is important to achieve anatomical reduction and stable internal fixation for excellent functional outcome.

\section{ACKNOWLEDGMENT}

We thank our department staff for their support. We thank Dr. M S Latha for editing and proofreading.

\section{AUTHORS' CONTRIBUTIONS}

Dr Krishna Bhargava Vem provided the design, intellectual content, innovations, and protocol design for the study. Dr Shiva Kumar Challa contributed the study design and protocol design, interpreted the results, and prepared the manuscript. Prof D Srinivas Murthy approved the study design, supervised the conduct of the study including assessments, patient follow-up, and data collection, reviewed, and approved the manuscript.

\section{CONFLICTS OF INTEREST}

The authors declare that there are no conflicts of interest regarding the publication of this article.

\section{REFERENCES}

1. Knight RA, Purvis GD. Fractures of both bones of the forearm in adults. J Bone Joint Surg Am 1949;31 A:755-64.

2. Reddy CC, Nazeer BS, Arun HS. A study of management of fractures of both bones forearm using locking compression plates. Int J Curr Res 2017;9:47593-8

3. Frigg R. Development of the locking compression plate. Injury 2003;34 Suppl 2:B6-10.

4. Leung F, Chow SP. A prospective, randomized trial comparing the limited contact dynamic compression plate with the point contact fixator for forearm fractures. J Bone Joint Surg Am 2003;85-A:2343-8.

5. Egol KA, Kubiak EN, Fulkerson E, Kummer FJ, Koval KJ. Biomechanics of locked plates and screws. J Orthop Trauma 2004;18:488-93.

6. Leung F, Chow SP. Locking compression plate in the treatment of forearm fractures: A prospective study. J Orthop Surg (Hong Kong) 2006;14:291-4.

7. Haidukewych GJ. Innovations in locking plate technology. J Am Acad Orthop Surg 2004;12:205-12

8. Dickson KF, Munz J. Locked plating: Clinical indications. Tech Orthop 2007;22:181-5.

9. Stevens CT, ten Duis HJ. Plate osteosynthesis of simple forearm fractures: LCP versus DC plates. Acta Orthop Belg 2008;74:180-3.

10. Meena RK, Singh AM, Langshong R, Waikhom S, Singh AK, Chishti SN. Internal fixation of adult diaphyseal both bone forearm fractures using locking compression plate. J Med Soc 2014;28:171-4.

11. Ranganath HD, Channappa TS, Suhas BD, Somashekhar S. A study of surgical management of fracture both bones forearm treated with limited contact dynamic compression plate and screws. Int J Orthop Sci 2017;3:785-91

12. Sharma S, Dang H, Sharma V, Sharma S. Treatment of diaphyseal forearm bone fractures by locking compression plate (LCP). Internet J Orthop Surg 2008;2008:11.

13. Reddy BJ, Lingala A, Kathyayini R. Comparative study of forearm fractures treated with locking compression plate limited contact dynamic compression plate. J Evol Med Dent Sci 2015;4:2001-10.

14. Saikia K, Bhuyan S, Bhattacharya T, Borgohain M, Jitesh P, Ahmed F, et al. Internal fixation of fractures of both bones forearm: Comparison of locked compression and limited contact dynamic compression plate. Indian J Orthop 2011;45:417-21.

15. Andrew HC. Fractures of shoulder girdle, arm and forearm. Ch. 49. In: Canale E, Tery S. Campbells Operative Orthopaedics. St. Louis, Mo: Mosby; 2013. P. 2829-916.

16. Perren SM. Basic aspect of internal fixation. Ch. 1. In: Muller ME, Allgower M, Scheider R, Willenegger H, editor. Manual of Internal Fixation. $3^{\text {rd }}$ ed. Berlin: Springer Verlag; 1991. P. 240-2.

17. Anderson LD, Sisk D, Tooms RE, Park WI $3^{\text {rd }}$. Compression-plate fixation in acute diaphyseal fractures of the radius and ulna. J Bone Joint Surg Am 1975;57:287-97

18. Chip ML. Forearm fractures. Ch. 14. In: Hausen ST, Swiontkowski MF. Orthopaedic Trauma Protocols. New York: Raven Press; 1993. p. 121-4.

19. Hansmann C. Eine neue möglichkeit der fixierung der frag- mente bei komplizierten frakturen. Verh dtsch Ges Chir 1886;3:158.

20. Sommer C, Gautier E, Müller M, Helfet DL, Wagner M. First clinical results of the locking compression plate (LCP). Injury 2003;34 Suppl 2:B43-54

21. Burwell HN, Charnley AD. Treatment of forearm fractures in adults with particular reference to plate fixation. J Bone Joint Surg Br 1964:46:404-25.

22. Moed BR, Kellam JF, Foster RJ, Tile M, Hansen ST Jr. Immediate internal fixation of open fractures of the diaphysis of the forearm. J Bone Joint Surg Am 1986;68:1008-17.

23. Herbert DS, Gerald CW. Treatment of fractures of the radius and ulna with compression plates: A retrospective study of one hundred and nineteen fractures in seventy-eight patients. JBJS 1972;54:1167-76.

24. Chapman MW, Gordon JE, Zissimos AG. Compression-plate fixation of acute fractures of the diaphyses of the radius and ulna. J Bone Joint Surg Am 1989;71:159-69.

25. Teipner WA, Mast JW. Internal fixation of forearm diaphyseal fractures: Double plating versus single compression (tension band) plating - a comparative study. Orthop Clin North Am 1980;11:381-91.

26. Grace TG, Eversmann WW Jr. Forearm fractures: Treatment by rigid 
fixation with early motion. J Bone Joint Surg Am 1980;62:433-8.

27. Smith JE. Internal fixation in the treatment of fractures of the shafts of the radius and ulna in adults; the value of delayed operation in the prevention of non-union. J Bone Joint Surg Br 1959;41-B:122-31.

28. Sarmiento A, Cooper JS, Sinclair WF. Forearm fractures. Early functional bracing - A preliminary report. J Bone Joint Surg Am 1975;57:297-304.

29. McKee MD, Seiler JG, Jupiter JB. The application of the limited contact dynamic compression plate in the upper extremity: An analysis of 114 consecutive cases. Injury 1995;26:661-6.

30. Prakash S, Basanthi BS. Study of functional outcome of fracture forearm bones by open reduction and lc-dcp fixation. Int J Curr Pharm Clin Res 2013;3:8-12.

31. Naik SR, Gunnaiah KG. Functional outcome of surgical management of fracture both bones forearm in adults using LC-DCP. J Evol Med Dent Sci 2014;3:11134-45

32. Muralidhar BM, Ravi KB, Madhusudan H. Surgical management of fracture both bone forearm in adult using limited contact dynamic compression plate. Int J Orthop Sci 2017;3:852-6.

33. Azboy I, Demirtas A, Uçar BY, Bulut M, Alemdar C, Ozkul E, et al. Effectiveness of locking versus dynamic compression plates for diaphyseal forearm fractures. Orthopedics 2013;36:e917-22.

34. Niemeyer P, Südkamp NP. Principles and clinical application of the locking compression plate (LCP). Acta Chir Orthop Traumatol Cech 2006; 73:221-8.

35. Sommer C, Babst R, Müller M, Hanson B. Locking compression plate loosening and plate breakage: A report of four cases. J Orthop Trauma 2004; 18:571-7. 